\title{
Secrecy Analysis of NOMA-Based Integrated Satellite-Multiple UAV Networks under Nonideal Hardware and Colluding Scheme
}

\author{
Yuanyuan Wang $\mathbb{D}^{1},{ }^{1}$ Feng Zhou $\left(\mathbb{D},{ }^{1}\right.$ Rugang Wang $\mathbb{D}^{1},{ }^{1}$ Xueling Wang $\mathbb{D}^{2},{ }^{2}$ and Kefeng Guo $\mathbb{i D}^{3}$ \\ ${ }^{1}$ School of Information Technology, Yancheng Institute of Technology, Yancheng 224051, China \\ ${ }^{2}$ Space E-Star Communication Technology Co. Ltd., Nanjing 210007, China \\ ${ }^{3}$ Space Information School, Space Engineering University, Beijing 101417, China \\ Correspondence should be addressed to Kefeng Guo; guokefeng.cool@163.com
}

Received 25 December 2021; Revised 9 January 2022; Accepted 15 January 2022; Published 3 February 2022

Academic Editor: Xingwang Li

Copyright (c) 2022 Yuanyuan Wang et al. This is an open access article distributed under the Creative Commons Attribution License, which permits unrestricted use, distribution, and reproduction in any medium, provided the original work is properly cited.

\begin{abstract}
This paper studies the secrecy outage probability (SOP) of nonorthogonal multiple access (NOMA)-based integrated satellitemultiple unmanned aerial vehicle networks (ISMUAVNs) under nonideal hardware and colluding scheme. Colluding scheme is defined as that the eavesdroppers cooperate with each other to overhear the legitimate users' signals. On this foundation, we obtain the closed-form expression for the SOP for the considered NOMA-based ISMUAVNs along with the partial unmanned aerial vehicle (UAV) selection scheme. To gain more things in the high signal-to-noise ratio (SNR) regime, we derive the asymptotic expression for the SOP, which gives fast ways to evaluate the benefit of the NOMA scheme and the impact of nonideal hardware impairments on the SOP. Finally, Monte Carlo simulation results are provided to show the correctness of the analytical results. In addition, with the help of representative results, some important and interesting findings are obtained.
\end{abstract}

\section{Introduction}

The integration of satellite communication (SatCom) networks with the terrestrial mobile networks is one of the key elements for the fifth-generation (5G) and beyond 5G networks due to enhanced capability and extended coverage [1]. However, the coverage region may be restricted by the obstacles and shadowing between the terrestrial users and satellite, which leads to the unavailability of the line-of-sight (LOS) links [2]. Under this situation, the terrestrial relay is utilized to overcome this challenge such as fading, shadowing, and path loss [3], which leads to the formation of the integrated satellite-unmanned aerial vehicle networks (ISUAVNs). Particularly, the main idea of ISUAVNs is to utilize terrestrial relays/unmanned aerial vehicles (UAVs) to forward the satellite signal, which has been a good framework for the SatCom, they have great effects on the fixed satellite service and high-speed broadband mobile satellite service, and SiriusXM is the real system which relies on the foundation of this architecture [4].
As the wide coverage of the satellite beam, there are often many wireless communication nodes in one satellite beam, which result in the suitable selection scheme for the wireless communication nodes [1]. In [5], the outage probability (OP) was analyzed with the opportunistic wireless node scheduling scheme for the integrated satellite-terrestrial relay networks (ISTRNs). In [6], the OP was investigated for the ISTRNs under the consideration of cognitive technology and multiple terrestrial transmission nodes.

With the breakthrough of UAV technology, UAV communication has been widely utilized in many temporary large-scale activities and natural disasters [7]. Total power constraint-beamforming and per-antenna power constraintbeamforming schemes were proposed to optimize the energy efficiency of integrated satellite-UAV terrestrial networks in [7]. The authors of [8] investigated the UAV system with mobile edge computing, where the energy expenditure of users was minimized under assignment delay demands and resource limitations. In [9], the game theory was applied to optimize the resources' allocation of the UAV 
communication network for more rewards, and a multiagent reinforcement learning framework was proposed to find the optimal policy. The potential game, mean-field game, Stackelberg game, graphical game, and coalition game were utilized to solve the resource management problem of largescale UAVs in [10]. The authors of [11] designed a decaying deep Q-network-based algorithm to minimize the energy consumption, in which the trajectory of the UAV, phase shifts of the reconfigurable intelligent surfaces, power allocation strategy, and dynamic decoding sequence were considered.

To alleviate the spectrum shortage and meet the requirements of improved spectral efficiency of ISTRNs, nonorthogonal multiple access (NOMA) has been viewed as the new paradigm of multiple access techniques [12-14], which transmits signals simultaneously in the same time/ frequency resource block. On this foundation, successive interference cancellation (SIC) is used at the receivers to distinguish the users' message by different power levels [15]. In NOMA systems, the message for the weaker was first decoded by the stronger users in which case, the stronger can be regarded as the relay to help the user with poor channel environment to enhance the system performance [16, 17]. In [18], the energy efficiency, ergodic capacity, and outage probability (OP) of NOMA-based downlink SatCom were investigated. In [19], the cooperative transmission method was analyzed for the NOMA-based SatCom. In [15], the authors derived the closed-form expression of OP in the presence of secondary networks.

As presented before, the satellite beam has a wide coverage, which results in the security problem in reality for the ISTRNs [20]; physical layer security (PLS) is considered as a new method to present the security problem of the wireless transmission networks. The major idea of PLS is to keep the secrecy transmission rate in the case of no interception under malicious nodes. In [21], the authors listed enough problems in the SatCom and investigated the secrecy performance. In [22], the authors investigated the SOP and ASC with the maximum user scheduling scheme for the SatCom. In [23], the authors analyzed the impact of imperfect channel stat information (CSI) for SatCom with multiple legitimate users and multiple eavesdroppers. In $[24,25]$, the ASC and SOP for the ISTRNs with the opportunistic relaying scheme were analyzed, respectively.

It is mentioned that, in real wireless communication systems, the transmission nodes are often not ideal owing to several reasons [26, 27]. In [28], the authors summarized all the reasons and proposed a general nonideal hardware model to research the impact of nonideal hardware on the wireless communication networks. In [29], the authors investigated the performance for the amplify-and-forward multiple relay networks in the presence of nonideal hardware and available direct transmission link. In [30], the OP was analyzed with the nonideal hardware and cognitive technology existing in the ISTRNs.

Until now, the impact of nonideal hardware on the NOMA-based SatCom was only investigated in several former papers, such as [31-34]. In [31], the authors analyzed the SOP for the considered SatCom with nonideal hardware and channel estimation errors (CEEs). In [32], the impact of nonideal hardware was investigated for the ISTRNs. In [33], Guo analyzed the effect of nonideal hardware on the ISTRNs with multiple terrestrial relays under the partial wireless node selection scheme.

In this paper, we investigate the secrecy NOMA-based integrated satellite-multiple unmanned aerial vehicle networks (ISMUAVNs) in the presence of multiple eavesdroppers and HIs by utilizing the partial UAV selection scheme. In detail, the main contributions of this paper are shown in the following:

(i) Firstly, we establish a NOMA-based secrecy ISMUAVN with nonideal hardware. The partial UAV selection scheme with low implementation complexity is applied in the considered system.

(ii) Secondly, the closed-form expression for SOP of the considered network in the presence of nonideal hardware and the proposed UAV selection scheme is derived.

(iii) Finally, to get insight of the SOP at high signal-tonoise ratios (SNRs), the asymptotic expression for SOP of the considered secrecy ISMUAVNs is also derived.

The rest of this paper is given in the following. Section 2 introduces the system model and formulates the secrecy problem of the considered secrecy ISMUAVNs. Section 3 provides the detailed analysis for the SOP. In Section 3, the secrecy diversity order and secrecy coding gain are analyzed along with the asymptotic analysis. In Section 4, numerical Monte Carlo (MC) results are obtained. In Section 5, the summarization is presented.

1.1. Notations. $|\cdot|$ denotes the absolute value of a complex scalar, $\exp (\cdot)$ is the exponential function, $E[\cdot]$ represents the expectation operator, and $\mathscr{C} \mathscr{N}(\mathbf{a}, b)$ is the complex Gaussian distribution of a random vector a and covariance matrix $b$.

\section{System Model}

As plotted in Figure 1, in this paper, we investigate NOMAbased secrecy ISMUAVNs, which consist of a satellite $(S)$, namely, Alice, multiple UAVs $\left(R_{p}, p \in\{1, \ldots, M\}\right)$, two destinations (namely, $D_{1}$ and $D_{2}$ ), and $N$ eavesdroppers. Decode-and-forward (DF) protocol is utilized at $R$. Two time slots are used for the total transmission. The direct transmission link is not considered between $S$ and $D_{i} i \in\{1,2\} 29$, for some reasons (Owing to some practical reasons, such as heavy fading, shadowing, or obstacle blockage, no direct transmission link is assumed in this paper.). In general, all transmission nodes in the considered NOMA-based ISMUAVNs are equipped with one antenna, respectively (In this paper, only two users are considered; however, the derived results can also be suitable for multiple users, which will be investigated in the near future. In this paper, the single antenna is assumed; however, the derived results will also be suitable for the case with multiple antennas.). 


\section{SYSTEM MODEL}

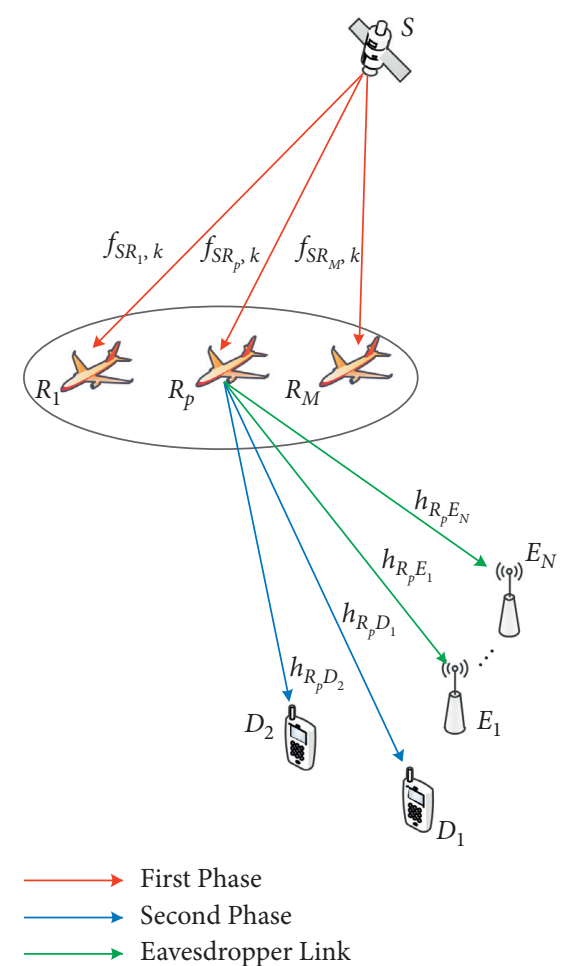

Figure 1: Illustration of the system model.

For the first time slot, the signal $s(t)$ is forwarded by $S$ with $E\left[|s(t)|^{2}\right]=1$ to $p$ th $R$. The received signal at $p$ th $R$ is derived as

$$
y_{R_{p}}(t)=f_{S R_{p}, k} \sqrt{P_{S}}\left[s(t)+\eta_{S}(t)\right]+n_{R_{p}}(t),
$$

where $f_{S R_{p}, k}$ represents the channel coefficient modeled as shadowed-Rician (SR) fading (In this paper, the perfect CSI is assumed, and our results will be as the branch for the case with imperfect CSI.) and $P_{S}$ denotes the transmit power from $S$. When applying the NOMA scheme, the signal is shown as $s(t)=a_{1 p} x_{1}(t)+a_{2 p} x_{2}(t)$, where $x_{1}(t)$ and $x_{2}(t)$ present the target transmitted signal to $D_{1}$ and $D_{2}$, respectively. Besides, it is assumed that $a_{1 p}>a_{2 p}$ for the reason that the channel $D_{1}$ suffers heavy fading than that of $D_{2}$. On this foundation, much power should be allocated to $D_{1} \cdot a_{1 p}$ and $a_{2 p}$ represent the transmission power allocation (PA) factors for $D_{1}$ and $D_{2}$, respectively. In addition, $a_{1 p}^{2}+a_{2 p}^{2}=1 . \eta_{S}(t)$ is the distortion noise due to nonideal hardware with $\eta_{S}(t) \sim C \mathcal{N}\left(0, a_{1 p}^{2} k_{1 p}^{2}+a_{2 p}^{2} k_{2 p}^{2}\right) . k_{1 p}$ and $k_{2 p}$ denote the nonideal levels for nonideal hardware which are defined in [3]. $n_{R_{f}}(t)$ represents the additive white Gaussian noise (AWGN) at $p$ th $R$ distributed as $n_{R_{p}}(t) \sim C \mathcal{N}\left(0, \delta_{R_{p}}^{2}\right)$.

When the signal is received by $R$, SIC works as follows: $x_{1}(t)$ is firstly decoded and then deleted from the obtained signal [12]. Thereafter, $x_{2}(t)$ is decoded from the left signal (In this paper, perfect SIC is assumed. Besides, different users are distinguished by the channel quality. We assume that the nearby user has a better channel quality than that of the distant users which has been assumed in the literature studies.).
Hence, the signal-to-interference-plus-noise-anddistortion ratio (SINDR) of detecting $x_{1}(t)$ at $p$ th $R$ is given by

$$
\gamma_{R_{p}, 1}=\frac{\gamma_{1 p} a_{1 p}^{2}}{\gamma_{1 p}\left(a_{1 p}^{2} k_{1 p}^{2}+a_{2 p}^{2}+a_{2 p}^{2} k_{2 p}^{2}\right)+1},
$$

where $\gamma_{1 p}=\left|f_{S R_{p}, k}\right|^{2} P_{S} / \delta_{R_{p}}^{2}$.

Moreover, we can obtain the signal-to-noise-and-distortion ratio (SNDR) of detecting $x_{2}(t)$ at $p$ th $R$ as

$$
\gamma_{R_{p}, 2}=\frac{\gamma_{1 p} a_{2 p}^{2}}{\gamma_{1 p}\left(a_{1 p}^{2} k_{1 p}^{2}+a_{2 p}^{2} k_{2 p}^{2}\right)+1} .
$$

As announced before, the partial UAV selection scheme [35] is adopted for the considered system model, and the SINDR of $x_{1}(t)$ and $x_{2}(t)$ during the first transmission link is, respectively, shown as

$$
\begin{aligned}
& \gamma_{R, 1}=\max _{p \in\{1, \ldots, M\}}\left(\gamma_{R_{p}, 1}\right), \\
& \gamma_{R, 2}=\max _{p \in\{1, \ldots, M\}}\left(\gamma_{R_{p}, 2}\right) .
\end{aligned}
$$

For the second time slot, the selected $p$ th $R$ forwards received signal $s(t)$ to $D_{1}$ and $D_{2}$, respectively; thus, the signal received at $D_{i}$ can be represented as

$y_{D_{p i}}(t)=h_{R_{p} D_{i}} \sqrt{P_{R_{p}}}\left(\xi_{1 p} x_{1}(t)+\xi_{2 p} x_{2}(t)+\eta_{p}(t)\right)+n_{D_{i}}(t)$,

where $h_{R_{p} D_{i}}$ denotes the channel coefficient between $p$ th $R$ and $D_{i}$ which undergoes Rayleigh fading, $P_{R_{p}}$ represents the transmit power for $p$ th $R, x_{i}(t)$ denotes the expected signal, $\xi_{j p} j \in\{1,2\}$ which suits $\xi_{1 p}>\xi_{2 p}$, and $\xi_{1 p}^{2}+\xi_{2 p}^{2}=1$ represents the PA factors at $p$ th $R$, respectively (We apply the same assumption which means the channel quality of user $D_{1}$ is worse than that of user $D_{2}$.). $n_{D_{i}}(t)$ denotes the AWGN at $D_{i}$ distributed as $n_{D_{i}}(t) \sim \mathscr{C} \mathscr{N}\left(0, \delta_{D_{i}}^{2}\right) . \quad \eta_{p}(t)$ with $\eta_{p}(t) \sim \mathscr{C} \mathcal{N}\left(0, \xi_{1 p}^{2} k_{p 1}^{2}+\xi_{2 p}^{2} k_{p 2}^{2}\right)$ represents the distortion noise which is caused by nonideal hardware. $\xi_{1 p}$ along with $\xi_{2 p}$ denotes the nonideal levels of nonideal hardware [35]. Therefore, $D_{2}$ performs SIC to decode $x_{1}(t)$ (while $x_{2}(t)$ is treated as the cochannel noise).

From (6), the SINDR of $x_{1}(t)$ sent by $p$ th $R$ at $D_{2}$ is represented as

$$
\gamma_{D_{p 1}, D_{2}}=\frac{\gamma_{2 p} \xi_{1 p}^{2}}{\gamma_{2 p}\left(\xi_{1 p}^{2} k_{1 p}^{2}+\xi_{2 p}^{2}+\xi_{2 p}^{2} k_{2 p}^{2}\right)+1},
$$

where $\gamma_{2 p}=\left|h_{R_{p} D_{2}}\right|^{2} P_{R_{p}} / \delta_{D_{2}}^{2}$.

$\gamma_{D_{p 1}, D_{2}}$ must be satisfied as $\gamma_{D_{p 1}, D_{2}} \geq \gamma_{t h}$ due to the same condition like (2). $D_{2}$ deleted the decoded information from the received signal by observing its own signal; hence, the final SNDR for signal $x_{2}(t)$ forwarded by $p$ th $R$ is given by 


$$
\gamma_{D_{p 2}, D_{2}}=\frac{\gamma_{2 p} \xi_{2 p}^{2}}{\gamma_{2 p}\left(\xi_{1 p}^{2} k_{1 p}^{2}+\xi_{2 p}^{2} k_{2 p}^{2}\right)+1} .
$$

Then, $x_{1}(t)$ is decoded by $D_{1}$, while $x_{2}(t)$ is regarded as the serial interference; the SINDR received at $D_{1}$ from $p$ th $R$ can be obtained as

$$
\gamma_{D_{p 1}, D_{1}}=\frac{\gamma_{3 p} \xi_{1 p}^{2}}{\gamma_{3 p}\left(\xi_{1 p}^{2} k_{1 p}^{2}+\xi_{2 p}^{2}+\xi_{2 p}^{2} k_{2 p}^{2}\right)+1}
$$

where $\gamma_{3 p}=\left|h_{R_{p} D_{1}}\right|^{2} P_{R_{p}} / \delta_{D_{1}}^{2}$.

At the same time, due to the fact that the signal sent to relays can be received by all eavesdroppers, the heard signal of the $j$ th eavesdropper can be represented as

$y_{E_{p j}}(t)=h_{R_{p} E_{j}} \sqrt{P_{R_{p}}}\left(\xi_{1 p} x_{1}(t)+\xi_{2 p} x_{2}(t)+\eta_{p}(t)\right)+n_{E_{j}}(t)$,

where $h_{R_{p} E_{j}}$ represents the channel coefficient between $p$ th $R$ and the $j$ th Eve which is modeled as Rayleigh fading. $n_{E_{i}}(t)$ with $n_{E_{j}}(t) \sim \mathscr{C} \mathcal{N}\left(0, \delta_{E_{j}}^{2}\right)$ denotes the AWGN at the $j$ th Eve.

Through the whole paper, we assume that parallel interference cancellation (PIC) is available at each eavesdropper [36, 37]. Hence, the received SNR of $i$ th D's expected messages at the $j$ th Eve can be shown as

$$
\lambda_{D_{p i} E_{j}}=\frac{\xi_{i p}^{2} \gamma_{D_{p i} E_{j}}}{\gamma_{D_{p i} E_{j}}\left(\xi_{1 p}^{2} k_{1 p}^{2}+\xi_{2 p}^{2} k_{2 p}^{2}\right)+1},
$$

where $\gamma_{D_{p i} E_{j}}=\left|h_{R_{p} E_{j}}\right|^{2} P_{R_{p}} / \delta_{E_{j}}^{2}$.

Due to the cooperation of all eavesdroppers, we can get the SNR of all Eves' link as

$$
\gamma_{D_{p i} E}=\sum_{j=1}^{N} \lambda_{D_{p i} E_{j}}
$$

The authors in [1] introduced the definition of the secrecy capacity; with the help of (7), (8), (9), and (12), we can obtain the secrecy capacity for the $i$ th signal as

$$
C_{S_{p i} \rho}=\left[C_{B_{p i} \rho}-C_{D_{p i} E}\right]^{+},\left(\rho \in\left\{D_{1}, D_{2}\right\}\right),
$$

where $[x]^{+} \stackrel{\Delta}{=} \max [x, 0], C_{B_{p i} \rho}=\log _{2}\left(1+\gamma_{D_{p i}, \rho}\right)$, and $C_{D_{p i} E}=$ $\log _{2}\left(1+\gamma_{D_{p i} E}\right)$ (CBpi means the secrecy capacity at the $\rho$ user to detect the $i$ th signal from $p$ th $R$.).

\section{Performance Analysis}

\subsection{The Channel Model}

3.1.1. Satellite Channel Model. As mentioned before, the satellite-UAV links are considered to be modeled as SR fading, so the probability density function (PDF) of $\gamma_{1 p}$ can be shown as

$$
f_{\gamma_{1 p}}(x)=\frac{\alpha_{1 p}}{\bar{\gamma}_{1 p}} e^{-\beta_{1} / \bar{\gamma}_{1}}{ }_{1} F_{1}\left(m_{1 p} ; 1 ; \frac{\delta_{1 p}}{\bar{\gamma}_{1 p}} x\right), x>0,
$$

where ${ }_{1} F_{1}(a ; b ; x)$ denotes the confluent hypergeometric function [38]. $\bar{\gamma}_{1 p}$ represents the average SNR between Alice and $p$ th $R, \quad \alpha_{1 p}=\left(2 b_{1 p} m_{1 p} / 2 b_{1 p} m_{1 p}+\Omega_{1 p}\right)^{m_{1 p} / 2 b_{1 p}}$, $\beta_{1 p}=1 / 2 b_{1 p}$, and $\delta_{1 p}=\Omega_{1 p} / 2 b_{1 p}\left(2 b_{1 p} m_{1 p}+\Omega_{1 p}\right) \quad$ with $m_{1 p} \geq 0,2 b_{1 p}$, and $\Omega_{1 p}$ being the fading severity parameter ranging from 0 to $\infty$, the average power of the multipath component, and the average power of the LOS component, respectively. In this paper, we assume that $m_{1 p}$ is an integer, and the PDF and cumulative distribution function (CDF) of $\gamma_{1 p}$ are, respectively, written as

$$
\begin{aligned}
& f_{\gamma_{1 p}}(x)=\alpha_{1 p} \sum_{k_{1}=0}^{m_{1 p}-1} \frac{\left(1-m_{1 p}\right)_{k_{1}}\left(-\delta_{1 p}\right)^{k_{1}}}{\left(k_{1} !\right)^{2} \bar{\gamma}_{1 p}^{k_{1}+1}} x^{k_{1}} \exp \left(-\Delta_{1 p} x\right), \\
& F_{\gamma_{1 p}}(x)=1-\alpha_{1 p} \sum_{k_{1}=0}^{m_{1 p}-1} \sum_{t=0}^{k_{1}} \frac{\left(1-m_{1 p}\right)_{k_{1}}\left(-\delta_{1 p}\right)^{k_{1}}}{k_{1} !\left(\bar{\gamma}_{1 p}\right)^{k_{1}+1} t ! \Delta_{1 p}^{k_{1}-t+1}} x^{t} e^{-\Delta_{1 p} x},
\end{aligned}
$$

where $\Delta_{1 p}=\beta_{1 p}-\delta_{1 p} / \bar{\gamma}_{1 p}$ and $(\cdot)_{k}$ denotes the Pochhammer symbol [38].

3.1.2. Terrestrial Channel Model. In the former section, we assume the channel model between $R$ and intended users or eavesdroppers which undergoes independent and identically distributed (i.i.d) Rayleigh fading. Thus, the CDF and PDF of $\gamma_{U}\left(U \in\left\{2 p, 3 p, D_{i} E_{j}\right\}\right)$ are, respectively, shown as

$$
F_{\gamma_{U}}=1-e^{-x / \bar{\gamma}_{U}}
$$

and

$$
f_{\gamma_{U}}=\frac{1}{\bar{\gamma}_{U}} e^{-x / \bar{\gamma}_{U}},
$$

where $\bar{\gamma}_{U}$ is the average channel gain.

By utilizing (11) and (12) along with [5], the CDF and PDF of $\gamma_{D_{p i} E}$ are, respectively, derived as 


$$
\begin{aligned}
F_{\gamma_{D_{p i} E}}(x)= & 1-\sum_{v=0}^{N-1} \frac{1}{v}\left[\frac{x}{\bar{\gamma}_{D_{p i} E} \xi_{i p}^{2}-x \bar{\gamma}_{D_{p i} E}\left(\xi_{1 p}^{2} k_{1 p}^{2}+\xi_{2 p}^{2} k_{2 p}^{2}\right)}\right]^{v} e^{-x / \bar{\gamma}_{D_{p i} E}\left[\xi_{i p}^{2}-x\left(\xi_{1 p}^{2} k_{1 p}^{2}+\xi_{2 p}^{2} k_{2 p}^{2}\right)\right]} \\
f_{\gamma_{D_{p i} E}}(x)= & \frac{\xi_{1 p}^{2} \bar{\gamma}_{D_{p i} E}^{-N}}{\left[\xi_{i p}^{2}-x\left(\xi_{1 p}^{2} k_{1 p}^{2}+\xi_{2 p}^{2} k_{2 p}^{2}\right)\right]^{2}(N-1) !}\left(\frac{x}{\bar{\gamma}_{D_{p i} E} \xi_{i p}^{2}-x \bar{\gamma}_{D_{p i} E}\left(\xi_{1 p}^{2} k_{1 p}^{2}+\xi_{2 p}^{2} k_{2 p}^{2}\right)}\right) \\
& \times \exp \left(-\frac{x}{\bar{\gamma}_{D_{p i} E}\left[\xi_{i p}^{2}-x\left(\xi_{1 p}^{2} k_{1 p}^{2}+\xi_{2 p}^{2} k_{2 p}^{2}\right)\right]}\right) .
\end{aligned}
$$

3.2. Secrecy Outage Probability. SOP refers to the probability of reaching the nonnegative target confidentiality rate. In this section, the SOP of the NOMA-based ISMUAVN describes the confidentiality behavior, and it can be expressed as

$$
P_{\text {out }}\left(\gamma_{0}\right)=P_{1}\left(\gamma_{0}\right)+P_{2 D_{2}}\left(\gamma_{0}\right)-P_{1}\left(\gamma_{0}\right) P_{2 D_{2}}\left(\gamma_{0}\right)
$$

where

$$
P_{1}\left(\gamma_{0}\right)=P_{1 D_{1}}\left(\gamma_{0}\right)+P_{1 D_{2}}\left(\gamma_{0}\right)-P_{1 D_{1}}\left(\gamma_{0}\right) P_{1 D_{2}}\left(\gamma_{0}\right)
$$

and

$$
P_{2 D_{2}}\left(\gamma_{0}\right)=\mathrm{P}_{12 D_{2}}\left(\gamma_{0}\right)+\mathrm{P}_{22 D_{2}}\left(\gamma_{0}\right)-\mathrm{P}_{12 D_{2}}\left(\gamma_{0}\right) \mathrm{P}_{22 D_{2}}\left(\gamma_{0}\right),
$$

where

$$
P_{1 \rho}\left(\gamma_{0}\right)=\mathrm{P}_{11 \rho}\left(\gamma_{0}\right)+\mathrm{P}_{21 \rho}\left(\gamma_{0}\right)-\mathrm{P}_{11 \rho}\left(\gamma_{0}\right) \mathrm{P}_{21 \rho}\left(\gamma_{0}\right)
$$

By using (4) and (5), $P_{1 i \rho}$ and $P_{2 i \rho}$ are, respectively, presented as

$$
\begin{aligned}
& P_{1 i \rho}\left(\gamma_{0}\right)=\left[\operatorname{Pr}\left(\gamma_{R_{p}, i} \leq \gamma_{0}\right)\right]^{M}, \quad i \in\{1,2\} \\
& P_{2 i \rho}\left(\gamma_{0}\right)=\operatorname{Pr}\left(C_{S_{p i \rho}} \leq C_{0}\right)=\int_{0}^{\infty} \int_{0}^{\gamma_{0}+y\left(1+\gamma_{0}\right)} f_{\gamma_{D_{p i}, \rho}}(x) f_{\gamma_{D_{p i} E}}(y) \mathrm{d} x \mathrm{~d} y .
\end{aligned}
$$

Also, $C_{0}=\log _{2}\left(1+\gamma_{0}\right)$.

$$
P_{1 D_{1}}\left(\gamma_{0}\right)=P_{11 D_{1}}\left(\gamma_{0}\right)+P_{21 D_{1}}\left(\gamma_{0}\right)-P_{11 D_{1}}\left(\gamma_{0}\right) P_{21 D_{1}}\left(\gamma_{0}\right) \text {, }
$$

In the following, to make the analysis easier, $\bar{\gamma}_{D_{p 1} E}=$ $\bar{\gamma}_{D_{p^{2}} E}=\bar{\gamma}_{E}$ is assumed, and the detailed analysis of SOP for $D_{1}$ and $D_{2}$ is, respectively, given in the following theorems.

where

Theorem 1. At $D_{1}$, the closed-form expression for $P_{1 D_{1}}$ by detecting $x_{1}(t)$ is given by

$$
P_{11 D_{1}}\left(\gamma_{0}\right)=\left[1-\sum_{k_{1}=0}^{m_{1 p}-1} \sum_{t=0}^{k_{1}} \frac{\alpha_{1 p}\left(1-m_{1 p}\right)_{k_{1}}\left(-\delta_{1 p}\right)^{k_{1}}}{k_{1} !\left(\bar{\gamma}_{1 p}\right)^{k_{1}+1} t ! \Delta_{1 p}^{k_{1}-t+1}} \times e^{-\Delta_{1 p} \gamma_{0} / a_{1 p}^{2}-\gamma_{0} B}\left(\frac{\gamma_{0}}{a_{1 p}^{2}-\gamma_{0} B}\right)^{t}\right]^{M}
$$

and

In (25), $B=a_{1 p}^{2} k_{1 p}^{2}+a_{2 p}^{2}+a_{2 p}^{2} k_{2 p}^{2}$. In (26),

$$
P_{21 D_{1}}\left(\gamma_{0}\right)=1-\frac{H_{1}}{2 \bar{\gamma}_{E}^{2 N-1}(N-1) !} \sum_{i=1}^{K_{1}} w_{i} H_{1}\left(z_{i}\right)
$$

$$
H_{1}(z)=\frac{\xi_{1 p}^{2} z^{N-1}}{\left(\xi_{1 p}^{2}-z C\right)^{N+1}} \exp \left(-\frac{z}{\xi_{1 p}^{2} \bar{\gamma}_{E}\left(\xi_{1 p}^{2}-z C\right)}\right) \exp \left\{-\frac{\gamma_{0}+z\left(1+\gamma_{0}\right)}{\bar{\gamma}_{3 p} \xi_{1 p}^{2}-\left[\gamma_{0}+z\left(1+\gamma_{0}\right)\right] \bar{\gamma}_{3 p} A}\right\}
$$


where $K_{1}$ denotes the number of terms, $H_{1}=\min \left(\xi_{1 p}^{2} / C\right.$, $\left.\left(\xi_{1 p}^{2}-A \gamma_{0}\right) /\left(A+A \gamma_{0}\right)\right), \quad A=\xi_{1 p}^{2} k_{1 p}^{2}+\xi_{2 p}^{2}+\xi_{2 p}^{2} k_{2 p}^{2}$, $C=\xi_{1 p}^{2} k_{1 p}^{2}+\xi_{2 p}^{2} k_{2 p}^{2}, z_{i}=t_{i}+1$ presents the $i$ th zero of Legendre polynomials, and $w_{i}$ denotes the Gaussian weight, which is given in Table (25.4) of [39].

With the similar method, at $D_{2}$, the exact expression of $P_{1 D_{2}}$ to decode $x_{1}(t)$ can be expressed as

$$
P_{1 D_{2}}\left(\gamma_{0}\right)=P_{11 D_{2}}\left(\gamma_{0}\right)+P_{21 D_{2}}\left(\gamma_{0}\right)-P_{11 D_{2}}\left(\gamma_{0}\right) P_{21 D_{2}}\left(\gamma_{0}\right) \text {, }
$$

where $P_{11 D_{2}}\left(\gamma_{0}\right)=P_{11 D_{1}}\left(\gamma_{0}\right)$,

$$
P_{21 D_{2}}\left(\gamma_{0}\right)=1-\frac{H_{2}}{2 \bar{\gamma}_{E}^{2 N-1}(N-1) !} \sum_{i=1}^{K_{2}} w_{i} H_{2}\left(z_{i}\right)
$$

$$
H_{2}(z)=\frac{\xi_{1 p}^{2} z^{N-1}}{\left(\xi_{1 p}^{2}-z C\right)^{N+1}} \exp \left(-\frac{z}{\xi_{1 p}^{2} \bar{\gamma}_{E}\left(\xi_{1 p}^{2}-z C\right)}\right) \exp \left\{-\frac{\gamma_{0}+z\left(1+\gamma_{0}\right)}{\bar{\gamma}_{2 p} \xi_{1 p}^{2}-\left[\gamma_{0}+z\left(1+\gamma_{0}\right)\right] \bar{\gamma}_{2 p} A}\right\}
$$

where $H_{2}=\min \left(\xi_{1 p}^{2} / C,\left(\xi_{1 p}^{2}-A \gamma_{0}\right) /\left(A+\gamma_{0} A\right)\right)$.

$$
P_{2 D_{2}}\left(\gamma_{0}\right)=P_{12 D_{2}}\left(\gamma_{0}\right)+P_{22 D_{2}}\left(\gamma_{0}\right)-P_{12 D_{2}}\left(\gamma_{0}\right) P_{22 D_{2}}\left(\gamma_{0}\right),
$$

Proof. See Appendix A.

where

Theorem 2. $x_{2}(t)$ is only detected by $D_{2}$; therefore, the exact expression of $P_{2 D_{2}}$ to decode $x_{1}(t)$ can be represented as

$$
P_{12 D_{2}}\left(\gamma_{0}\right)=\left[1-\sum_{k_{1}=0}^{m_{1 p}-1} \sum_{t=0}^{k_{1}} \frac{\alpha_{1 p}\left(1-m_{1 p}\right)_{k_{1}}\left(-\delta_{1 p}\right)^{k_{1}}}{k_{1} !\left(\bar{\gamma}_{1 p}\right)^{k_{1}+1} t ! \Delta_{1 p}^{k_{1}-t+1}} \times e^{-\Delta_{1 p} \gamma_{0} / a_{2 p}^{2}-\gamma_{0} C}\left(\frac{\gamma_{0}}{a_{2 p}^{2}-\gamma_{0} C}\right)^{t}\right]^{M}
$$

and

where

$$
P_{22 D_{2}}\left(\gamma_{0}\right)=1-\frac{H_{3}}{2 \bar{\gamma}_{E}^{2 N-1}(N-1) !} \sum_{i=1}^{K_{3}} w_{i} H_{3}\left(z_{i}\right)
$$

$$
H_{3}(z)=\frac{\xi_{2 p}^{2} z^{N-1}}{\left(\xi_{2 p}^{2}-z C\right)^{N+1}} \exp \left(-\frac{z}{\xi_{2 p}^{2} \bar{\gamma}_{E}\left(\xi_{2 p}^{2}-z C\right)}-\frac{\gamma_{0}+z\left(1+\gamma_{0}\right)}{\bar{\gamma}_{2 p} \xi_{2 p}^{2}-\left[\gamma_{0}+z\left(1+\gamma_{0}\right)\right] \bar{\gamma}_{2 p} C}\right)
$$

Also, $H_{3}=\min \left(\xi_{2 p}^{2} / C,\left(\xi_{2 p}^{2}-C \gamma_{0}\right) /\left(C+\gamma_{0} C\right)\right)$.

Proof. See Appendix B.

3.3. Asymptotic SOP. In the behind description, to derive deeper insights of the impact of the significant system parameters of the considered NOMA-based ISMUAVN in the high SNR regime, we show the following analysis. According to (16) and (15), when $\bar{\gamma}_{1 p} \longrightarrow \infty$, we can derive

$$
F_{\gamma_{1 p}}(x) \approx \frac{\alpha_{1 p}}{\bar{\gamma}_{1 p}} x+o(x),
$$

where $o(x)$ denotes the infinitesimal of $x$ in the higher order. Similarly, when $\bar{\gamma}_{U} \longrightarrow \infty$, (17) can be rewritten as

$$
F_{\gamma_{U}}(x) \approx \frac{x}{\bar{\gamma}_{U}}+o(x)
$$

Proposition 1. At $D_{1}$, the asymptotic SOP for decoding $x_{1}(t)$ of our proposed system is represented as

$$
\begin{aligned}
P_{1 D_{1}}^{\infty}\left(\gamma_{0}\right)= & +\left[\frac{\alpha_{1 p}}{\bar{\gamma}_{1 p}}\left(\frac{\gamma_{0}}{a_{1 p}^{2}-\gamma_{0} B}\right)\right]^{M}-\sum_{i=1}^{K_{1}} \frac{z_{i}^{N-1} H_{1} w_{i} \xi_{1 p}^{2}}{2 \bar{\gamma}_{E}^{2 N-1}(N-1) !} \\
& \left.\times \frac{e^{-z_{i} / \xi_{1 p}^{2} \bar{\gamma}_{E}\left(\xi_{1 p}^{2}-z_{i} C\right)}}{\left(1-\frac{\gamma_{0}+z_{i}\left(1+\gamma_{0}\right)}{\left.\xi_{1 p}^{2}-z_{i} C\right)^{N+1}}\left(1 \bar{\gamma}_{3 p}^{2} \xi_{1 p}^{2}-\left[\gamma_{0}+z_{i}\left(1+\gamma_{0}\right)\right] \bar{\gamma}_{3 p} A\right.\right.}\right) .
\end{aligned}
$$

At $D_{2}$, the asymptotic SOP for decoding $x_{1}(t)$ of our proposed system is obtained as 


$$
\begin{aligned}
P_{1 D_{2}}^{\infty}\left(\gamma_{0}\right)= & 1+\left[\frac{\alpha_{1 p}}{\bar{\gamma}_{1 p}}\left(\frac{\gamma_{0}}{a_{1 p}^{2}-\gamma_{0} B}\right)\right]^{M}-\sum_{i=1}^{K_{2}} \frac{z_{i}^{N-1} H_{2} w_{i} \xi_{1 p}^{2}}{2 \bar{\gamma}_{E}^{2 N-1}(N-1) !} \\
& \times \frac{e^{-z_{i} / \xi_{1 p}^{2} \bar{\gamma}_{E}\left(\xi_{1 p}^{2}-z_{i} C\right)}}{\left(\xi_{1 p}^{2}-z_{i} C\right)^{N+1}}\left(1-\frac{\gamma_{0}+z_{i}\left(1+\gamma_{0}\right)}{\bar{\gamma}_{2 p} \xi_{1 p}^{2}-\left[\gamma_{0}+z_{i}\left(1+\gamma_{0}\right)\right] \bar{\gamma}_{2 p} A}\right) .
\end{aligned}
$$

$x_{2}(t)$ is only detected by $D_{2}$; therefore, the asymptotic expression of SOP for decoding $x_{2}(t)$ for our proposed system is shown as

$$
\begin{aligned}
P_{2 D_{2}}^{\infty}\left(\gamma_{0}\right)= & 1+\left[\frac{\alpha_{1 p}}{\bar{\gamma}_{1 p}}\left(\frac{\gamma_{0}}{a_{2 p}^{2}-\gamma_{0} C}\right)\right]^{M}-\sum_{i=1}^{K_{3}} \frac{z_{i}^{N-1} H_{3} w_{i} \xi_{2 p}^{2}}{2 \bar{\gamma}_{E}^{2 N-1}(N-1) !} \\
& \times \frac{e^{-z_{i} \xi_{2 p}^{2} \bar{\gamma}_{E}\left(\xi_{2 p}^{2}-z_{i} C\right)}}{\left(\xi_{2 p}^{2}-z_{i} C\right)^{N+1}}\left(1-\frac{\gamma_{0}+z_{i}\left(1+\gamma_{0}\right)}{\bar{\gamma}_{2 p} \xi_{2 p}^{2}-\left[\gamma_{0}+z_{i}\left(1+\gamma_{0}\right)\right] \bar{\gamma}_{2 p} C}\right) .
\end{aligned}
$$

Proof. At first, by replacing (15) and (16) with (35) and (36) and then with the same method of Appendixes A and B, the exact equations for the asymptotic analysis (namely, (37), (38), and (40)) are derived.

In this sequel, we obtain the diversity order along with the coding gain. By letting $\bar{\gamma}_{1 p}=\bar{\gamma}_{2 p}=\bar{\gamma}_{3 p}=\bar{\gamma}$, (22) can be expressed as

$$
P_{\text {out }}^{\infty}\left(\gamma_{0}\right) \approx G_{a}\left(\frac{1}{\bar{\gamma}}\right)^{G_{d}},
$$

where $G_{d}=\min (M, 1)$ denotes the secrecy diversity order, and the secrecy coding gain of the considered NOMA-based ISMUAVN includes two cases, i.e., when $M \leq 1$,

$$
G_{a}=\left\{\frac{2 \alpha_{1 p} \gamma_{0}}{a_{1 p}^{2}-\gamma_{0} B}+\frac{\alpha_{1 p} \gamma_{0}}{a_{2 p}^{2}-\gamma_{0} C}+2 \sum_{i=1}^{K_{1}} \frac{z_{i}^{N-1} H_{1} w_{i} \xi_{1 p}^{2} e^{-z_{i} / \xi_{1}^{2}} \bar{\gamma}_{E}\left(\xi_{1 p}^{2}-z_{i} C\right)}{2_{E}^{2-1}(N-1) !\left(\xi_{1 p}^{2}-z_{i} C\right)^{N+1}\left\{\xi_{1 p}^{2}-\left[\gamma_{0}+z_{i}\left(1+\gamma_{0}\right)\right] A\right\}} \quad+\sum_{i=1}^{K_{3}} \frac{z_{i}^{N-1} H_{3} w_{i} \xi_{2 p}^{2} e^{-z_{i} / \xi_{2 p}^{2} \bar{\gamma}_{E}\left(\xi_{2 p}^{2}-z_{i} C\right)}\left[\gamma_{0}+z_{i}\left(1+\gamma_{0}\right)\right]}{2-1) !\left(\xi_{2 p}^{2}-z_{i} C\right)^{N-1}\left\{\xi_{2 p}^{2}-\left[\gamma_{0}+z_{i}\left(1+\gamma_{0}\right)\right] C\right\}} .\right.
$$

When $M>1$,

$$
G_{a}=\left\{2 \sum_{i=1}^{K_{1}} \frac{z_{i}^{N-1} H_{1} w_{i} \xi_{1 p}^{2} e^{-z_{i} / \xi_{1 p}^{2} \bar{\gamma}_{E}\left(\xi_{1 p}^{2}-z_{i} C\right)}\left[\gamma_{0}+z_{i}\left(1+\gamma_{0}\right)\right]}{2 \bar{\gamma}_{E}^{2-1}(N-1) !\left(\xi_{1 p}^{2}-z_{i} C\right)^{N+1}\left\{\xi_{1 p}^{2}-\left[\gamma_{0}+z_{i}\left(1+\gamma_{0}\right)\right] A\right\}}+\sum_{i=1}^{K_{3}} \frac{z_{i}^{N-1} H_{3} w_{i} \xi_{2 p}^{2} e^{-z_{i} i \xi_{2 p}^{2} \bar{p}_{E}\left(\xi_{2 p}^{2}-z_{i} C\right)}\left[\gamma_{0}+z_{i}\left(1+\gamma_{0}\right)\right]}{2-1(N-1) !\left(\xi_{2 p}^{2}-z_{i} C\right)^{N-1}\left\{\xi_{2 p}^{2}-\left[\gamma_{0}+z_{i}\left(1+\gamma_{0}\right)\right] C\right\}} .\right.
$$

\section{Numerical Results}

The correctness of the performance analysis of our considered system is verified by Monte Carlo simulations. In general, we assume $\delta_{R}^{2}=\delta_{D_{1}}^{2}=\delta_{D_{2}}^{2}=\delta_{E_{j}}^{2}=1, K=M=8$, and $\bar{\gamma}_{1 p}=\bar{\gamma}_{2 p}=\bar{\gamma}_{3 p}=\bar{\gamma}$. The channel parameters are given in Table 1 [5]. Different power coefficient combinations are assumed as follows: scenario 1: $a_{1}^{2}=\xi_{1}^{2}=0.9$ and $a_{2}^{2}=\xi_{2}^{2}=0.1$; scenario $2: a_{1}^{2}=\xi_{1}^{2}=0.8$ and $a_{2}^{2}=\xi_{2}^{2}=0.2$; scenario $3: a_{1}^{2}=\xi_{1}^{2}=0.7$ and $a_{2}^{2}=\xi_{2}^{2}=0.3$ (In this paper, the fixed power allocation factors are considered; however, the results derived can also be utilized for our future work when power allocation is studied.).

As proved in [40], a satellite channel model is expected to be general and applicable for a wide range of elevation angles, under which the satellite can be observed. In this regard, the most common approach to evaluate the impact of elevation angle on channel statistical parameters is based on the transformation from empirical expression. According to Abdi et al. [40], the maximum elevation angle is around $80^{\circ}$, while the minimum elevation angle is considered usually around $20^{\circ}$ in order to tackle the geographical terrain affects. Hence, this is particularly useful when we apply a set of data parameters with moderate variation to a model with specific shadowing and infrequent light shadowing conditions (i.e., frequent heavy shadowing, average shadowing, and infrequent light shadowing). Please note that, for specific shadowing conditions, different parameters have been employed to cover a range of elevation angles in many existing works [5].

Figure 2 illustrates the SOP versus $\bar{\gamma}$ and $k$ by setting $\bar{\gamma}_{E}=1 \mathrm{~dB}, \gamma_{0}=0 \mathrm{~dB}, N=2$, scenario 1 , and $M=3$ under different shadowing fading. We can find that when $\bar{\gamma}$ grows to be a fixed value, the SOP tends to be constant. Besides, it can be found that the SOP descends with the increase of the nonideal hardware level. In addition, it should be noted that the SOP under different channel conditions is consistent for high SNR regimes, which means that channel conditions will seriously affect the SOP.

Figure 3 plots the SOP versus $\bar{\gamma}$ for different $N$ and $k$ with $\gamma_{0}=0 \mathrm{~dB}, \bar{\gamma}_{E}=1 \mathrm{~dB}, M=3$, and scenario 1 under the AS scenario. It can be found that, with $N$ being larger, the SOP will be larger because more eavesdroppers are utilized to 
TABLE 1: Channel parameters.

\begin{tabular}{lccc}
\hline Shadowing & $m_{1 p}$ & $b_{1 p}$ & $\Omega_{1 p}$ \\
\hline Frequent heavy shadowing (FHS) & 1 & 0.063 & 0.0007 \\
Average shadowing (AS) & 5 & 0.251 & 0.279 \\
Infrequent light shadowing (ILS) & 10 & 0.158 & 1.29 \\
\hline
\end{tabular}
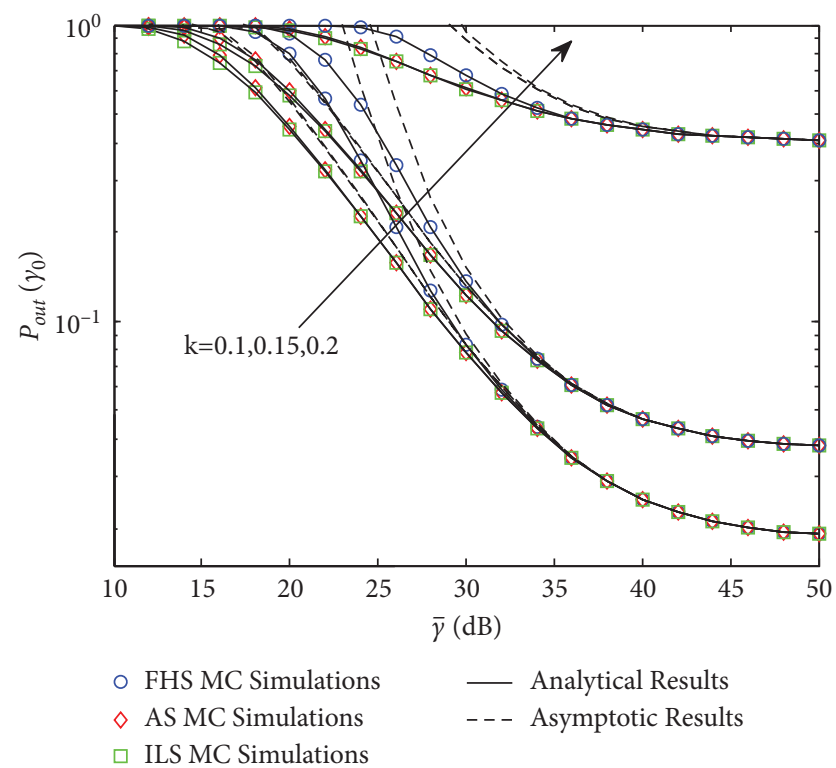

Figure 2: The SOP versus $\bar{\gamma}$ and $k$ by setting $\bar{\gamma}_{E}=1 \mathrm{~dB}, \gamma_{0}=0 \mathrm{~dB}$, $N=2$, scenario 1 , and $M=3$ under different shadowing fading.

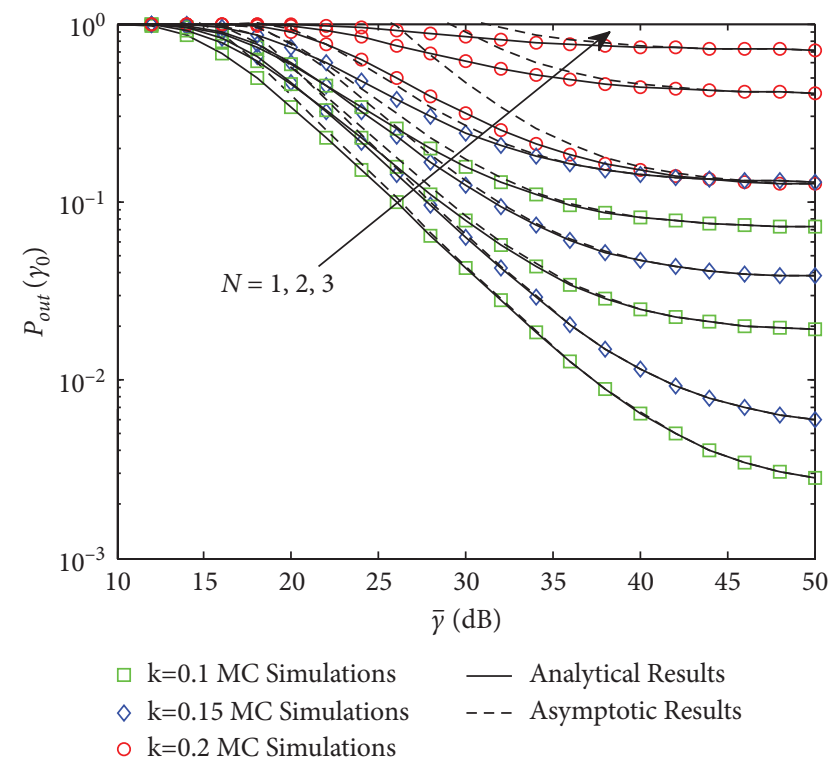

Figure 3: The SOP versus $\bar{\gamma}$ for different $N$ and $k$ with $\gamma_{0}=0 \mathrm{~dB}$, $\bar{\gamma}_{E}=1 \mathrm{~dB}, M=3$, and scenario 1 under the AS scenario.

steal the legitimate signals. Similar to Figure 2, the SOP will be a fixed value for big enough $\bar{\gamma}$. This is due to the fact that
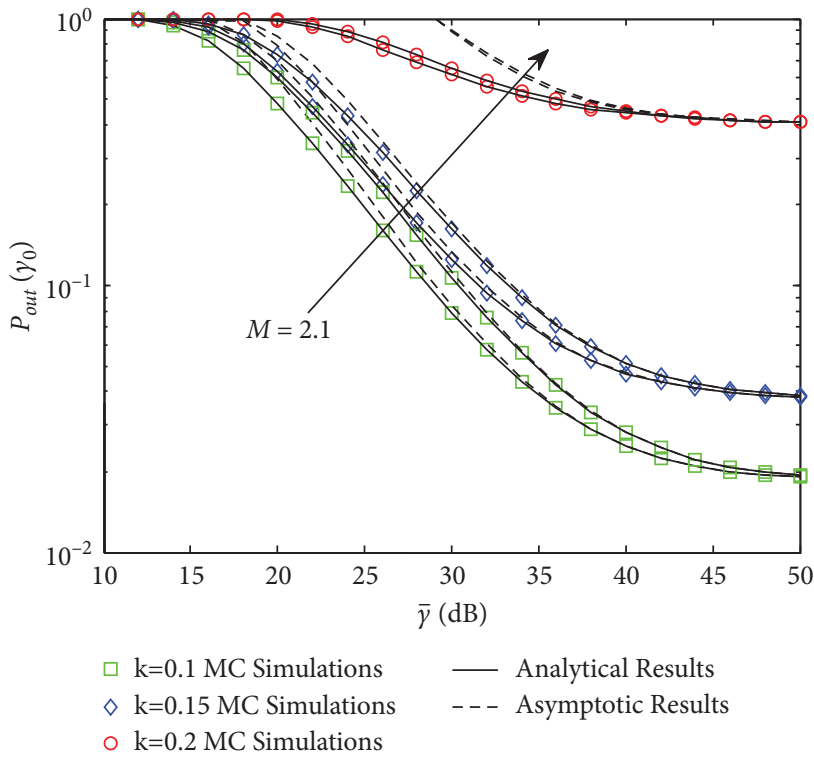

Figure 4: The SOP versus $\bar{\gamma}$ for different $M$ and $k$ with $\bar{\gamma}_{E}=1 \mathrm{~dB}$, $\gamma_{0}=0 \mathrm{~dB}, N=2$, and scenario 1 under the AS scenario.

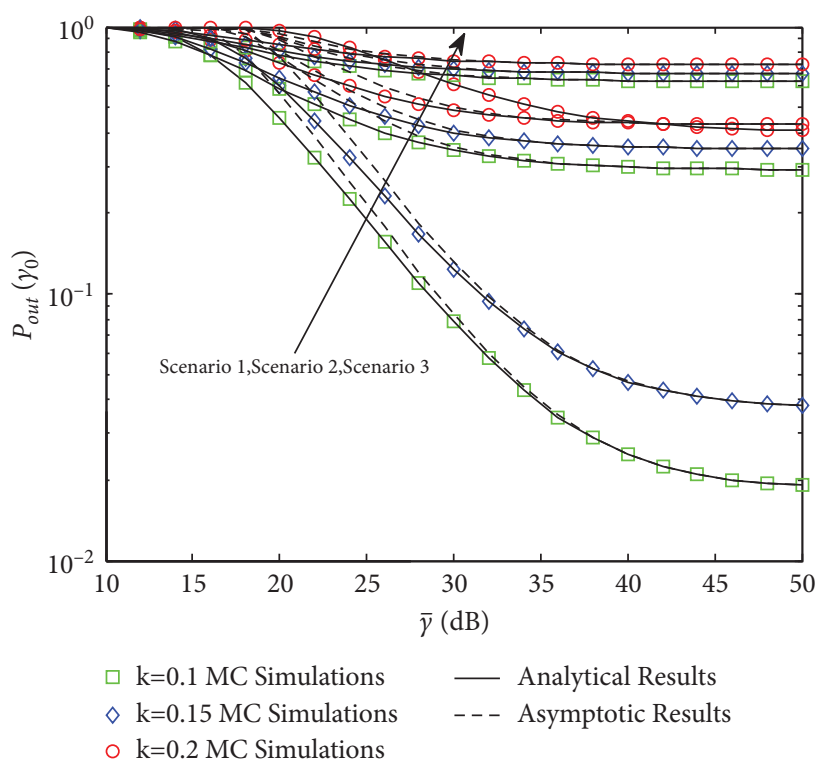

FIgURE 5: The SOP versus $\bar{\gamma}$ for different scenarios and $k$ with $\bar{\gamma}_{E}=1 \mathrm{~dB}, \gamma_{0}=0 \mathrm{~dB}, N=2$, and $M=3$ under the AS scenario.

an upper bound of the SINR is existed to the legitimate user, which is related to (2), (3), (7), (8), and (9).

Figure 4 examines the SOP versus $\bar{\gamma}$ for different $M$ and $k$ with $\bar{\gamma}_{E}=1 \mathrm{~dB}, \gamma_{0}=0 \mathrm{~dB}, N=2$, and scenario 1 under the AS scenario. We may obtain that when $M$ grows larger, the SOP will be lower. Figure 5 illustrates the SOP versus $\bar{\gamma}$ for different scenarios and $k$ with $\bar{\gamma}_{E}=1 \mathrm{~dB}, \gamma_{0}=0 \mathrm{~dB}, N=2$, and $M=3$ under the AS scenario. We can obtain that, with the gap of the power allocation factors becoming larger, the SOP will be lower; this is because the communication quality of the worse user is ensured. At last, we find that the secrecy 
performance will deteriorate with the nonideal hardware level.

\section{Conclusions}

In this paper, we studied the secrecy outage probability of NOMA-based ISMUAVNs in which nonideal hardware is considered and the partial UAV selection scheme is adopted. The exact closed-form expression of SOP for our proposed system was derived. Moreover, in the high SNR regime, we derived the asymptotic SOP. To get more insights, the secrecy coding gain and secrecy diversity order were obtained. The impacts of NOMA technology, nonideal hardware, and the partial UAV selection scheme on the performance of the considered system were revealed by theoretical analysis.

\section{Abbreviations}

AS:

Average shadowing

ASC:

Average secrecy capacity

AWGN:

Additive white Gaussian noise

CDF: $\quad$ Cumulative distribution function

CSI:

DF:

5G:

FHS:

Channel state information

Decode and forward

Fifth generation

ILS:

Frequency heavy shadowing

Infrequent light shadowing

ISMUAVNs: Integrated satellite-multiple unmanned aerial vehicle networks

ISTRNs: Integrated satellite-terrestrial relay networks

LOS: $\quad$ Line of sight

MC: $\quad$ Monte Carlo

NOMA: Nonorthogonal multiple access

OP: $\quad$ Outage probability

PDF: $\quad$ Probability density function

PIC: $\quad$ Parallel interference cancellation

PLS: $\quad$ Physical layer security

SatCom: Satellite communication

SIC: $\quad$ Successive interference cancellation
SINDR: Signal-to-interference-plus-noise-anddistribution ratio

SNDR: $\quad$ Signal-to-noise-and-distortion ratio

SNR: $\quad$ Signal-to-noise ratio

SNRs: $\quad$ Signal-to-noise ratios

SOP: $\quad$ Secrecy outage probability

SR: $\quad$ Shadowed-Rician

UAV: $\quad$ Unmanned aerial vehicle.

\section{Appendix}

\section{A. Proof of Theorem 1}

By utilizing (22), to get $P_{11 D_{1}}$, we should first derive $P_{11 D_{1}}\left(\gamma_{0}\right)$ and $P_{21 D_{1}}\left(\gamma_{0}\right)$. In the following, they are derived.

By utilizing (25) and (2), (25) is rewritten as

$P_{11 D_{1}}\left(\gamma_{0}\right)=\operatorname{Pr}\left[\max _{p \in\{1, \ldots, M\}}\left(\gamma_{R_{p}, 1}\right) \leq \gamma_{0}\right]=\left[F_{\gamma_{1 p}}\left(\frac{\gamma_{0}}{a_{1 p}^{2}-\gamma_{0} B}\right)\right]^{M}$.

From (23) and after some mathematical derivations, it can be seen that

$$
\begin{aligned}
\mathrm{P}_{21 D_{1}}\left(\gamma_{0}\right)= & \int_{0}^{H_{1}} F_{\gamma_{D_{p 1}, D_{1}}}\left(\gamma_{0}+y\left(1+\gamma_{0}\right)\right) f_{\gamma_{D_{p 1} E}}(y) \mathrm{d} y \\
& +\int_{H_{1}}^{\infty} f_{\gamma_{D_{p 1} E}}(y) \mathrm{d} y .
\end{aligned}
$$

In (A.2), obtaining the CDF of $\gamma_{D_{p 1}, D_{1}}$ and PDF of $\gamma_{D_{p 1} E}$ is the earlier thing needed to be done.

By utilizing (9) and (16), the CDF of $\gamma_{D_{p 1}, D_{1}}$ can be written as

$$
F_{\gamma_{D_{p 1}, D_{1}}}(x)=1-e^{\left.-\gamma_{0}+y\left(1+\gamma_{0}\right)\right) \bar{\gamma}_{3 p} \xi_{1 p}^{2}-\left[\gamma_{0}+y\left(1+\gamma_{0}\right)\right] \bar{\gamma}_{3 p} A} .
$$

By inserting (A.3) and (18) into (A.2), we can obtain

$$
\begin{aligned}
P_{21 D_{1}}\left(\gamma_{0}\right) & =\int_{0}^{\infty} \frac{\xi_{1 p}^{2} e^{-y / \xi_{1 p}^{2} \bar{\gamma}_{E}\left(\xi_{1 p}^{2}-y C\right)}\left\{1-e^{-\gamma_{0}+y\left(1+\gamma_{0}\right) / \bar{\gamma}_{3 p} \xi_{1 p}^{2}-\left[\gamma_{0}+y\left(1+\gamma_{0}\right)\right] \bar{\gamma}_{3 p} A}\right\}}{\bar{\gamma}_{E}^{N} y^{1-N}(N-1) !\left(\xi_{1 p}^{2}-y C\right)\left(\bar{\gamma}_{E} \xi_{1 p}^{2}-y \bar{\gamma}_{E} C\right)^{N-1}} \mathrm{~d} y \\
& =1-\frac{\bar{\gamma}_{E}^{-N} \xi_{1 p}^{2}}{(N-1) !}[\begin{array}{c}
\int_{0}^{H_{1}} \frac{e^{-\gamma_{0}+y\left(1+\gamma_{0}\right) / \bar{\gamma}_{3 p} \xi_{1 p}^{2}-\left[\gamma_{0}+y\left(1+\gamma_{0}\right)\right] \bar{\gamma}_{3 p} A}\left(\bar{\gamma}_{E} \xi_{1 p}^{2}-y \bar{\gamma}_{E} C\right)^{1-N}}{e^{y / \xi_{1 p}^{2} \bar{\gamma}_{E}\left(\xi_{1 p}^{2}-y C\right)} y^{1-N}\left(\xi_{1 p}^{2}-y C\right)} \mathrm{d} y \\
+\int_{H_{1}}^{\infty} \frac{e^{-y / \xi_{1 p}^{2} \bar{\gamma}_{E}\left(\xi_{1 p}^{2}-y C\right)} y^{N-1}}{\left(\xi_{1 p}^{2}-y C\right)\left(\bar{\gamma}_{E} \xi_{1 p}^{2}-y \bar{\gamma}_{E} C\right)^{N-1}} \mathrm{~d} y
\end{array} \underbrace{}_{H_{1}(y)}
\end{aligned}
$$

However, trying the authors' best, the exact expression of $H_{1}(\cdot)$ is difficult to be obtained. Thus, by utilizing the Gaussian-Chebyshev quadrature [39], $H_{1}(\cdot)$ is rewritten as
(27). By inserting (27) into (26) and after the simple process, we can finally derive the exact expression for $P_{21 D_{1}}\left(\gamma_{0}\right)$. Utilizing the similar methods, the exact 
expression for $P_{21 D_{2}}\left(\gamma_{0}\right)$ can also be obtained. The proof is completed.

\section{B. Proof of Theorem 2}

With the help of (8) and (16), the CDF of $\gamma_{D_{p 2}, D_{2}}$ can be expressed as

$$
F_{\gamma_{D_{p 2}, D_{2}}}(x)=1-e^{-\gamma_{0}+z\left(1+\gamma_{0}\right) / \bar{\gamma}_{2 p} \xi_{2 p}^{2}-\left[\gamma_{0}+z\left(1+\gamma_{0}\right)\right] \bar{\gamma}_{2 p} C} .
$$

Then, utilizing (3) and (15), the exact expression of $\mathrm{P}_{12 D_{2}}\left(\gamma_{0}\right)$ will be derived. Then, by inserting (B.1) and (18) into (23), we can obtain the exact expression of $\mathrm{P}_{22 D_{2}}\left(\gamma_{0}\right)$.

\section{Data Availability}

No data were used to support this study.

\section{Conflicts of Interest}

The authors declare that they have no conflicts of interest regarding the publication of this article.

\section{Acknowledgments}

This work was supported by the Natural Science Foundation of China (Grant nos. 61673108 and 62001517), in part by the Colleges and Universities Natural Science Foundation in Jiangsu Province (Grant no. 19KJA110002), and in part by the Industry-University-Research Cooperation Project of Jiangsu Province (nos. BY2020335 and BY2020358). Thanks go to all the authors for their contribution to this paper.

\section{References}

[1] K. Guo, K. An, B. Zhang et al., "Physical layer security for multiuser satellite communication systems with thresholdbased scheduling scheme," IEEE Transactions on Vehicular Technology, vol. 69, no. 5, pp. 5129-5141, 2020.

[2] K. Guo, K. An, F. Zhou, T. A. Tsiftsis, G. Zheng, and S. Chatzinotas, "On the secrecy performance of NOMA-based integrated satellite multiple-terrestrial relay networks with hardware impairments," IEEE Transactions on Vehicular Technology, vol. 70, no. 4, pp. 3661-3676, 2021.

[3] K. Guo, M. Lin, B. Zhang, W.-P. Zhu, J.-B. Wang, and T. A. Tsiftsis, "On the performance of LMS communication with hardware impairments and interference," IEEE Transactions on Communications, vol. 67, no. 2, pp. 1490-1505, 2019.

[4] K. Guo, M. Lin, B. Zhang et al., "Performance analysis of hybrid satellite-terrestrial cooperative networks with relay selection," IEEE Transactions on Vehicular Technology, vol. 69, no. 8, pp. 9053-9067, 2020.

[5] P. K. Sharma, P. K. Upadhyay, D. B. da Costa, P. S. Bithas, and A. G. Kanatas, "Performance analysis of overlay spectrum sharing in hybrid satellite-terrestrial systems with secondary network selection," IEEE Transactions on Wireless Communications, vol. 16, no. 10, pp. 6586-6601, 2017.

[6] K. Guo, K. An, B. Zhang, Y. Huang, and D. Guo, "On the performance of cognitive satellite-terrestrial relay networks with channel estimation error and hardware impairments," Sensors, vol. 18, pp. 1-19, 2018.
[7] Q. Huang, M. Lin, J.-B. Wang, T. A. Tsiftsis, and J. Wang, "Energy efficient beamforming schemes for satellite-aerialterrestrial networks," IEEE Transactions on Communications, vol. 68, no. 6, pp. 3863-3875, 2020.

[8] Y. Qu, H. Dai, H. Wang et al., "Service provisioning for UAVenabled mobile edge computing," IEEE Journal on Selected Areas in Communications, vol. 39, no. 11, pp. 3287-3305, 2021.

[9] J. Cui, Y. Liu, and A. Nallanathan, "Multi-agent reinforcement learning-based resource allocation for UAV networks," IEEE Transactions on Wireless Communications, vol. 19, no. 2, pp. 729-743, 2020.

[10] J. Chen, P. Chen, Q. Wu, Y. Xu, N. Qi, and T. Fang, “A gametheoretic perspective on resource management for large-scale UAV communication networks," China Communications, vol. 18, no. 1, pp. 70-87, 2021.

[11] X. Liu, Y. Liu, and Y. Chen, "Machine learning empowered trajectory and passive beamforming design in UAV-RIS wireless networks," IEEE Journal on Selected Areas in Communications, vol. 39, no. 7, pp. 2042-2055, 2021.

[12] S. M. R. Islam, M. Zeng, O. A. Dobre, and K.-S. Kwak, "Resource allocation for downlink NOMA systems: key techniques and open issues," IEEE Wireless Communications, vol. 25, no. 2, pp. 40-47, 2018.

[13] X. Li, "Cognitive amBC-NOMA IoV-MTS networks with IQI: reliability and security analysis," IEEE Transactions on Intelligent Transportation Systems, pp. 1-12, 2021.

[14] X. Li, M. Zhao, M. Zeng et al., "Hardware impaired ambient backscatter NOMA systems: reliability and security," IEEE Transactions on Communications, vol. 69, no. 4, pp. 27232736, 2021.

[15] R. Liu, K. Guo, K. An, S. Zhu, and H. Shuai, "NOMA-based integrated satellite-terrestrial relay networks under spectrum sharing environment," IEEE Wireless Communications Letters, vol. 10, no. 6, pp. 1266-1270, 2021.

[16] H. Lei, Z. Yang, K.-H. Park et al., "Secrecy outage analysis for cooperative NOMA systems with relay selection schemes," IEEE Transactions on Communications, vol. 67, no. 9, pp. 6282-6298, 2019.

[17] F. Zhou, Y. Wu, Y.-C. Liang, Z. Li, Y. Wang, and K.-K. Wong, "State of the art, taxonomy, and open issues on cognitive radio networks with NOMA," IEEE Wireless Communications, vol. 25, no. 2, pp. 100-108, 2018.

[18] X. Yan, H. Xiao, C.-X. Wang, K. An, A. T. Chronopoulos, and G. Zheng, "Performance analysis of NOMA-based land mobile satellite networks," IEEE Access, vol. 6, pp. 3132731339, 2018.

[19] R. Wan, L. Zhu, T. Li, and L. Bai, "A NOMA-PSO based cooperative transmission method in satellite communication systems," in Proceedings of the 2017 9th International Conference on Wireless Communications and Signal Processing (WCSP), vol. 2017, pp. 1-6, Nanjing, China, October 2017.

[20] K. Guo, K. An, B. Zhang, Y. Huang, and D. Guo, "Physical layer security for hybrid satellite terrestrial relay networks with joint relay selection and user scheduling," IEEE Access, vol. 6, pp. 55815-55827, 2018.

[21] B. Li, Z. Fei, C. Zhou, and Y. Zhang, "Physical-layer security in space information networks: a survey," IEEE Internet of Things Journal, vol. 7, no. 1, pp. 33-52, 2020.

[22] K. Guo, M. Lin, B. Zhang, J. Ouyang, and W.-P. Zhu, "Secrecy performance of satellite wiretap channels with multi-user opportunistic scheduling," IEEE Wireless Communications Letters, vol. 7, no. 6, pp. 1054-1057, 2018. 
[23] K. Guo, K. An, Y. Huang, and B. Zhang, "Physical layer security of multiuser satellite communication systems with channel estimation error and multiple eavesdroppers," IEEE Access, vol. 7, pp. 96253-96262, 2018.

[24] V. Bankey and P. K. Upadhyay, "Ergodic secrecy capacity analysis of multiuser hybrid satellite-terrestrial relay networks with multiple eavesdroppers," in Proceedings of the 2019 IEEE International Conference on Communications Workshops (ICC Workshops), pp. 1-6, Shanghai, China, May 2019.

[25] K. Guo, K. An, and X. Tang, "Secrecy performance for integrated satellite terrestrial relay systems with opportunistic scheme," in Proceedings of the 2019 IEEE International Conference on Communications Workshops (ICC Workshops), pp. 1-6, Shanghai, China, May 2019.

[26] X. Li, M. Huang, Y. Liu, V. G. Menon, A. Paul, and Z. Ding, "I/ $\mathrm{Q}$ imbalance aware nonlinear wireless-powered relaying of B5G networks: security and reliability analysis," IEEE Transactions on Network Science and Engineering, vol. 8, no. 4, pp. 2995-3008, 2021.

[27] X. Li, J. Li, Y. Liu, Z. Ding, and A. Nallanathan, "Residual transceiver hardware impairments on cooperative NOMA networks," IEEE Transactions on Wireless Communications, vol. 19, no. 1, pp. 680-695, 2020.

[28] E. Bjornson, M. Matthaiou, and M. Debbah, "A new look at dual-hop relaying: performance limits with hardware impairments," IEEE Transactions on Communications, vol. 61, no. 11, pp. 4512-4525, 2013.

[29] P. K. Sharma and P. K. Upadhyay, "Cognitive relaying with transceiver hardware impairments under interference constraints," IEEE Communications Letters, vol. 20, no. 4, pp. 820-823, 2016.

[30] K. Guo, K. An, B. Zhang, Y. Huang, and G. Zheng, "Outage analysis of cognitive hybrid satellite-terrestrial networks with hardware impairments and multi-primary users," IEEE Wireless Communications Letters, vol. 7, no. 5, pp. 816-819, 2018.

[31] F. Zhou, R. Wang, and K. Guo, "Secrecy outage analysis of satellite communication networks with hardware impairments and channel estimation errors," Electronics Letters, vol. 56, no. 20, pp. 1059-1062, 2020.

[32] X. Tang, K. An, K. Guo, Y. Huang, and S. A. Wang, "Outage analysis of non-orthogonal multiple access-based integrated satellite-terrestrial relay networks with hardware impairments," IEEE Access, vol. 7, pp. 141258-141267, 2019.

[33] K. Guo, "NOMA-based integrated satellite-terrestrial multirelay networks with hardware impairments and partial relay selections scheme," in Proceedings of the 2019 IEEE 19th International Conference on Communication Technology (ICCT), pp. 1-6, Xi'an, China, October 2019.

[34] F. Zhou, R. Wang, and J. Bian, "Performance analysis of nonorthogonal multiple access based-satellite communication networks with hardware impairments and channel estimations," Electronics Letters, vol. 56, no. 1, pp. 52-55, 2020.

[35] K. Guo, K. An, B. Zhang et al., "On the performance of the uplink satellite multiterrestrial relay networks with hardware impairments and interference," IEEE Systems Journal, vol. 13, no. 3, pp. 2297-2308, 2019.

[36] Z. Qin, Y. Liu, Z. Ding, Y. Gao, and M. Elkashlan, "Physical layer security for $5 \mathrm{G}$ non-orthogonal multiple access in largescale networks," in Proceedings of the 2016 IEEE International Conference on Communications (ICC), pp. 1-6, Kuala Lumpur, Malaysia, May 2016.

[37] Y. Liu, Z. Qin, M. Elkashlan, Y. Gao, and L. Hanzo, "Enhancing the physical layer security of non-orthogonal multiple access in large-scale networks," IEEE Transactions on Wireless Communications, vol. 16, no. 3, pp. 1656-1672, 2017.

[38] I. S. Gradshteyn, I. M. Ryzhik, A. Jeffrey, and D. Zwillinger, Table of Integrals, Series, and Products, Elsevier/Academic Press, Amsterdam, The Netherlands, 7th edition, 2007.

[39] M. Abramowitz and I. A. Stegun, Handbook of Mathematical Functions with Formulas, Graphs, and Mathematical Tables, Dover, Springfield, IL, USA, 9th edition, 1972.

[40] A. Abdi, W. C. Lau, M. Alouini, and M. Kaveh, "A new simple model for land mobile satellite channels: first- and secondorder statistics," IEEE Transactions on Wireless Communications, vol. 2, no. 3, pp. 519-528, 2003. 